\title{
Cutaneous involvement as the initial presentation of metastatic breast adenocarcinoma - Case report
}

\author{
Verônica Riquet de Siqueira ${ }^{1}$ \\ Israel Leitão Maia ${ }^{1}$ \\ Jose Telmo Valença Júnior ${ }^{1}$
}

\author{
Aline Salmito Frota ${ }^{1}$ \\ Hercília Maria Carvalho Queiroz ${ }^{1}$ \\ José Wilson Accioly Filho ${ }^{1}$
}

DOI: http://dx.doi.org/10.1590/abd1806-4841.20142995

\begin{abstract}
Cutaneous metastasis is an uncommon manifestation of visceral malignancy. It occurs late in the course of the disease but can be a sign of an unknown neoplasm. A case of local cutaneous metastasis by contiguity with breast adenocarcinoma is reported. It presented as initial manifestation and main complaint of a 68-year-old woman, with no family history and negative screening tests for the disease. Biopsies of ulcers which do not heal, persistent hardened erythema and cutaneous nodules of unknown cause must be performed, since the evidence of cutaneous metastasis can be of extreme importance for the diagnosis, staging and prognosis of an internal cancer. In this scenario, the dermatologist plays a fundamental role and should be always attentive to this diagnostic possibility.
\end{abstract}

Keywords: Breast neoplasms; Cutaneous neoplasms; Neoplastic metastasis

\section{INTRODUCTION}

Cutaneous metastasis is a relatively uncommon manifestation of visceral malignancy. ${ }^{1}$ Most of the times it occurs late in the course of the disease, but it can be the sign of presentation of an unknown neoplasm. It involves different dissemination ways (lymphatic, hematogenous, direct invasion of adjacent tissues and iatrogenic implantation); it may be present in the configuration of a widely disseminated disease, or be the only sign of metastatic involvement., ${ }^{2,3}$

A meta-analysis, which included studies of autopsies and retrospective studies, found a $5.3 \%$ global incidence of cutaneous metastasis in visceral neoplasms. ${ }^{1}$ However, it is not clearly defined in the literature if the prognosis differs in patients with exclusive cutaneous metastasis and in patients with visceral metastasis, with or without cutaneous involvement. ${ }^{3}$

Even though cutaneous metastases are rare, breast cancer is common in women and its metastasis frequently involves the skin, with cutaneous involvement in about one fourth of patients with the disease. . $^{1,5,56}$

\section{CASE REPORT}

Female patient, 68 years old, with onset of asymptomatic lesion in the intermammary region for about six months. She denied other complaints. At the physical examination she presented an erythematous infiltrated plaque of irregular surface, with central retraction, of hardened consistency, adhered to deep planes in the intermammary region (Figures 1 and 2). No other changes were shown in the rest of the physical examination.

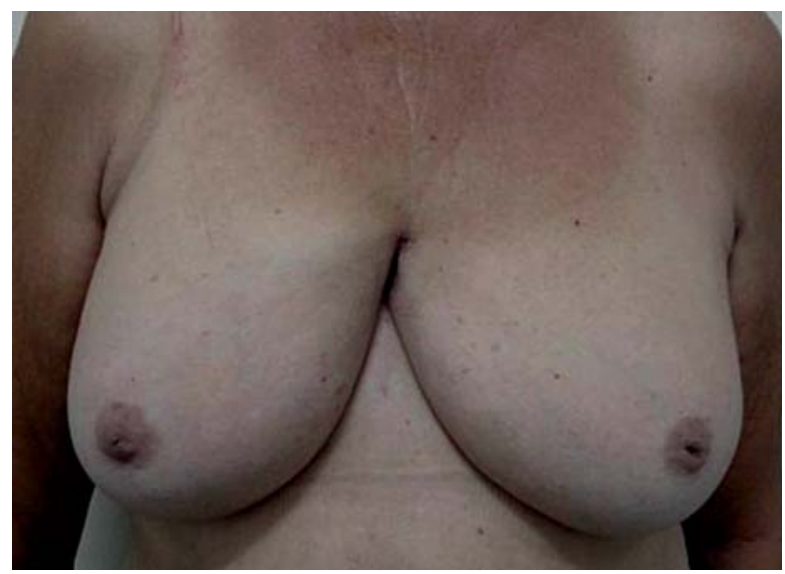

FIGURE 1: Lesion in retracted hardened plaque in intermammary region

\footnotetext{
Received on 21.07.2013.

Approved by the Advisory Board and accepted for publication on 15.10.2013.

* Work performed at Hospital Universitário Walter Cantídio - Universidade Federal do Ceará (HUWC-UFC) - Fortaleza (CE), Brazil.

Financial Support: none

Conflict of Interests: none

Universidade Federal do Ceará (UFC) - Fortaleza (CE), Brazil.
}

(C2014 by Anais Brasileiros de Dermatologia 


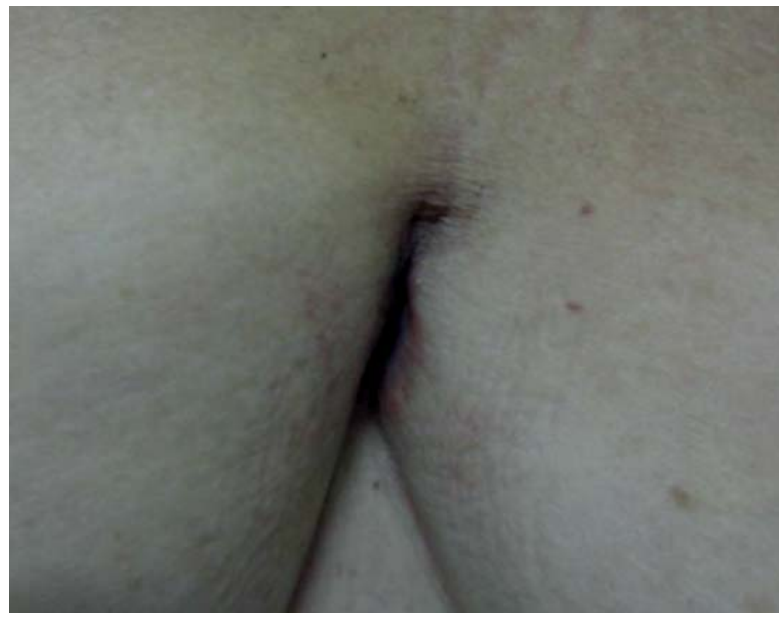

FIGURE 2: Detail of intermammary lesion evidencing erythema, infiltration and irregular surface

She reported positive smoking history for 37 years. She denied comorbidities or use of medications. Absence of mammary or lung cancer history in the family. Last preventative examination for cervical cancer and mammography were normal, performed less than one year ago.

Complete blood count and biochemistry laboratory exams, besides serum count of CA 15-3, CA 199, CA 125 as well as carcinoembryonic antigen were normal. Serology for HIV, viral hepatitides and syphilis were negative. Mammography and breast ultrasound evidenced only benign alterations, with BIRADS classification (Breast Imaging Reporting and Data System) II. Radiography and CT SCAN of thorax did not evidence significant changes. Multi-slice CT scan of abdomen and pelvis showed only focal parietal thickening of nonspecific aspect at the antrum/pylorus level. Thyroid and cervical region ultrasound images were normal.

Histopathologic examination of the cutaneous lesion revealed epidermis with no alterations. The dermis exhibited proliferation in blocks of atypical cells in a solid setting, other times similar to glands, irregular, with moderate nuclear pleomorphism and intense desmoplasia, compatible with poorly differentiated adenocarcinoma (Figures 3 and 4). Immunochemistry revealed diffuse positivity $(+++)$ in $90 \%$ of the neoplastic cells for estrogen receptors (ERs) and progesterone receptors (PRs), besides diffuse positivity for AE1/AE3, CK7 and mammoglobulin, focal positivity for $34 \mathrm{bE} 12$ and negativity for CDX 2 and TTF-1, being this immunohistochemical profile compatible with cutaneous infiltration by primary mammary adenocarcinoma (Figure 5).
Nuclear magnetic resonance (NMR) of breasts was performed, which demonstrated a spiculated nodular lesion in the right breast, medially, at the level of the intermammary cleft, measuring around 32×30 $\mathrm{mm}$, determining adjacent cutaneous retraction and thickening, with signs of invasion of adjacent thoracic wall, classified as BI-RADS 5 (Figure 6).

She was referred to the Clinical Oncology Service for better clinical staging, prognosis, and therapeutic follow-up. A new immunohistochemical study of the skin lesion was negative for c-erbB-2. Bone scintigraphy showed suggestive findings of secondary bone implants secondary to the base neoplasia in several studied sites. It was decided to start the radiotherapeutic treatment and use of tamoxifen $20 \mathrm{mg} /$ day. The patient is still being followed-up in conjunction with the Clinical Oncology Service.

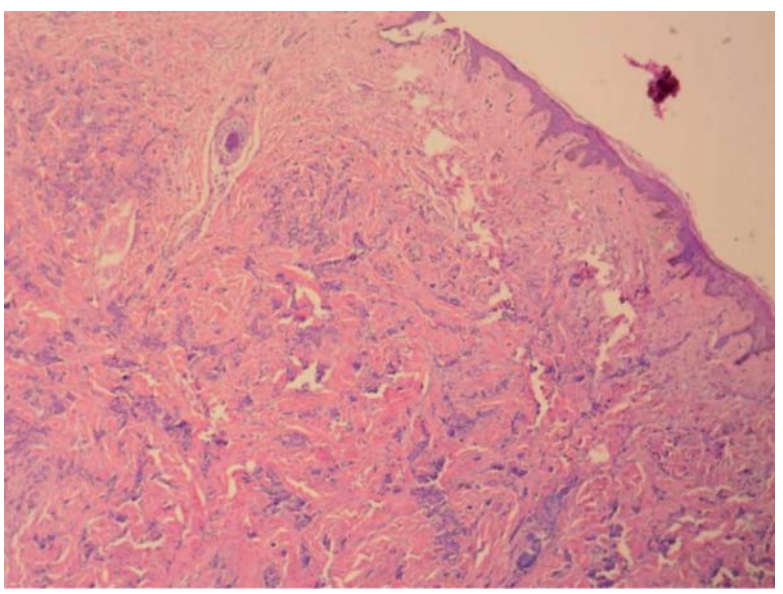

FIGURE 3: Histopathology of cutaneous lesion suggesting poorly differentiated adenocarcinoma

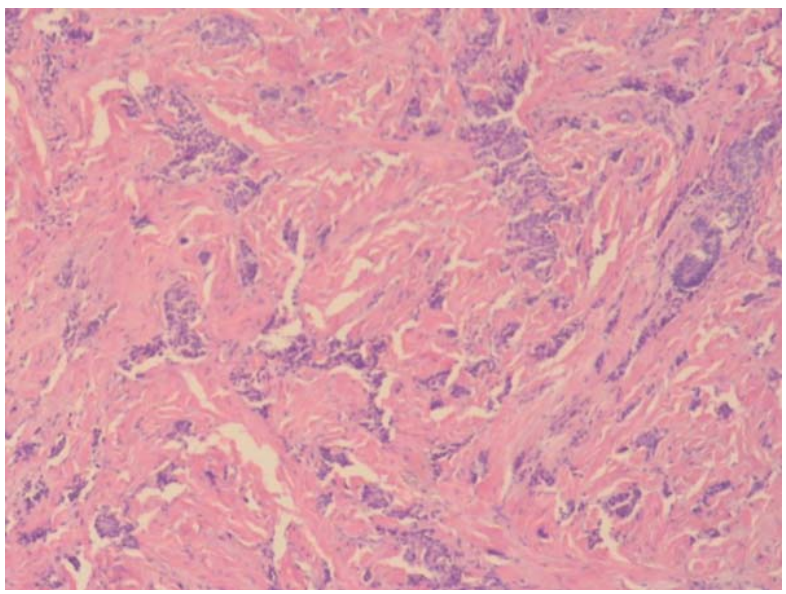

FIGURE 4: At the greatest magnification, HE showing blocks of atypical cells in a solid setting, at other times similar to glands, irregular, with moderate nuclear pleomorphism and intense desmoplasia 

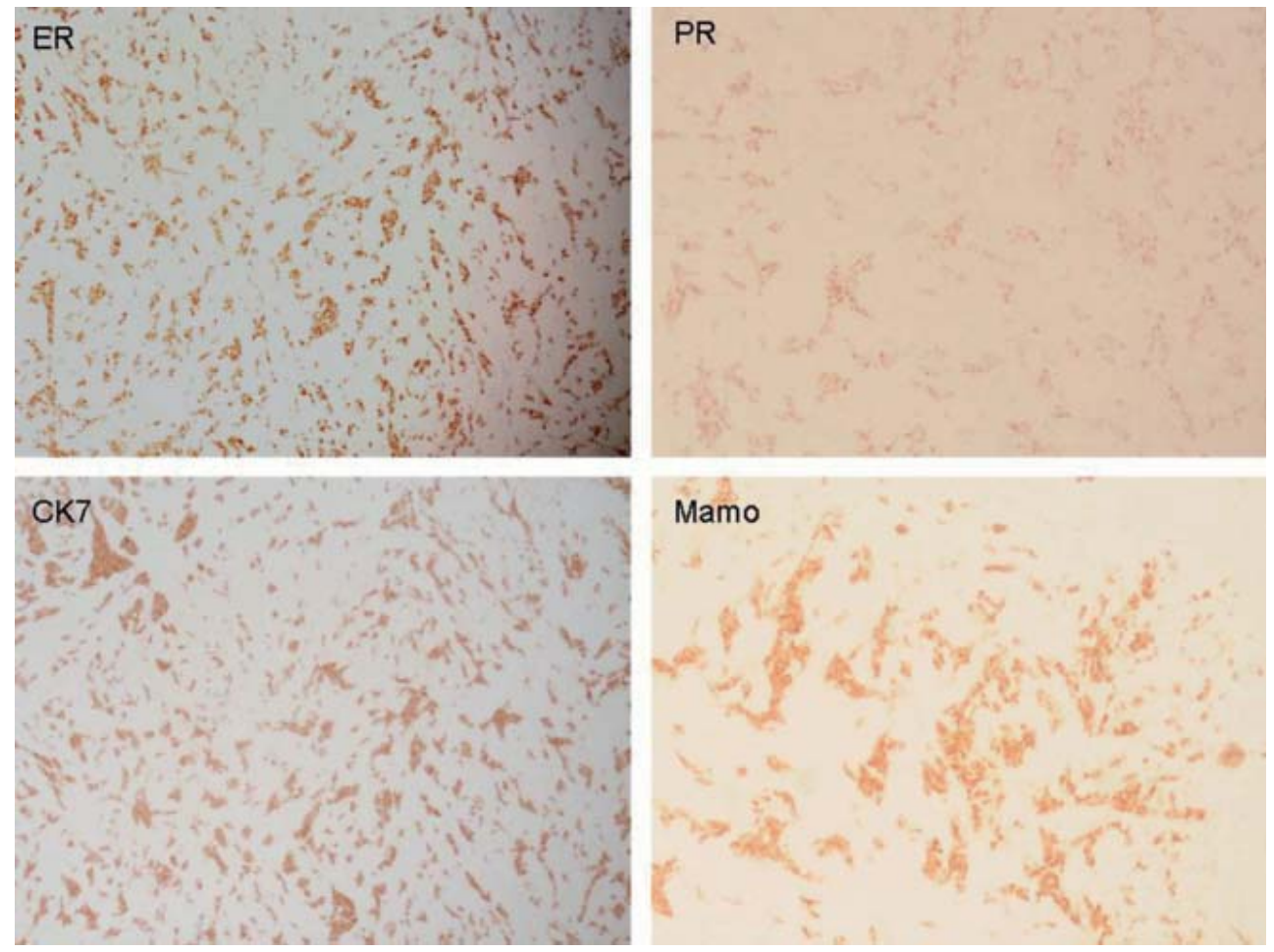

FIGURE 5:

Immunohistochemist ry of cutaneous lesion showing diffuse positivity for estrogen receptors (ER), progesterone receptors (PR), cytokeratin 7 (CK7) and Mammoglobulin (Mammo)

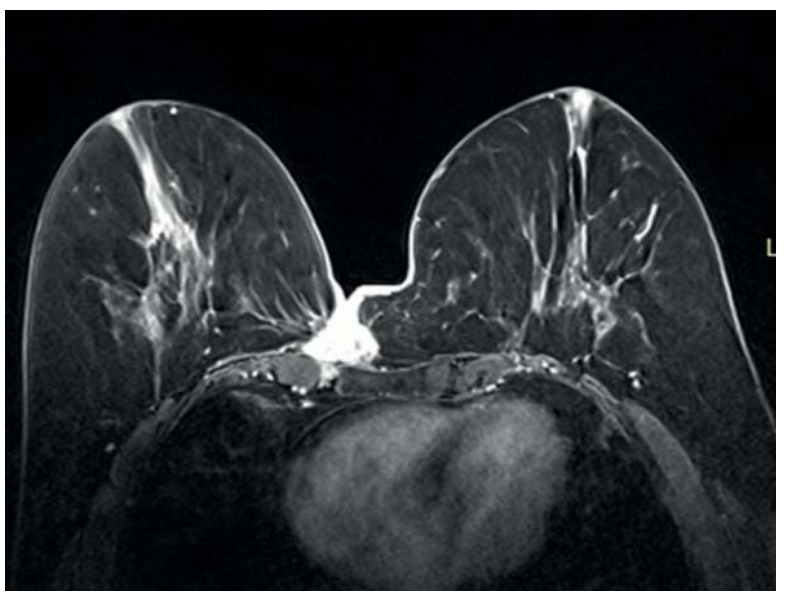

FIGURE 6: NMR of breasts, in T2, showing spiculated nodular lesion in the right breast. Contiguity of lesion with skin and invasion of subjacent thoracic wall is observed

\section{DISCUSSION}

In females, the most frequent primary site of cutaneous metastasis is, by far, the breast adenocarcinoma, mainly presenting with thorax involvement. ${ }^{1,4,5,7}$ The breast cancer cutaneous metastasis originates from a complex interaction between the tumor and skin, and can represent the first manifestation of the neoplasm. ${ }^{8}$ On the other hand, its detection can chan- ge the staging of the disease and, as a result, the prognosis and treatment of a patient with an already diagnosed breast cancer. ${ }^{8}$

A case of local cutaneous metastasis by contiguity to a breast adenocarcinoma in a 68-year-old woman, with no family history and presenting negative screening tests for the disease is reported here. The primary tumor was only revealed after a NMR of breasts. The case is even more relevant due to cutaneous metastasis being the initial sign and main complaint regarding the unknown neoplasm.

The carcinoma of breast has several distinct clinicopathological types of cutaneous involvement. ${ }^{6}$ Direct invasion of skin by tumor is manifested mainly by ulceration or inflammation. ${ }^{9}$ The patient in the studied case presented with a hardened, retracted and asymptomatic plaque lesion in the intermammary region. Histopathology and immunohistochemistry were essential for the diagnosis. Histologically, the cutaneous metastasis of breast cancer must be distinguished from a numerous variety of other neoplasms. ${ }^{8}$ The distinction between primary and metastatic adenocarcinomas of the skin is also important, due to the distinct clinical behavior of the two entities.

Regarding the finding about the cutaneous metastasis as the initial manifestation of the disease, it was verified that $6.3 \%$ of the patients with breast cancer presented skin involvement at the time of the pri- 
mary tumor diagnosis, and in 3.5\% skin involvement was the sign of the disease presentation. ${ }^{4}$

As for the prognosis, studies suggest differences in the prognosis of cutaneous metastases arising from different internal tumors, with patients suffering from breast cancer having a relatively better prognosis in comparison with other primary sites. ${ }^{3,5,10} \mathrm{~A}$ mean survival of 31 months after breast cancer diagnosis, with presence of cutaneous metastasis, was reported. ${ }^{5}$

Involvement of the skin occurs in internal cancer with some regularity, but its presence at diagnosis is not frequent, and even less frequent as representing the main complaint of the patient. ${ }^{4}$ However, surveillance must be maintained and biopsies of ulcers that do not heal, of persistent hardened erythema and of cutaneous nodules of undetermined causes must be performed for early detection of this cutaneous manifestation which, although uncommon, might be of extreme importance for the diagnosis, staging and prognosis of an internal cancer. ${ }^{4}$ In this scenario, the dermatologist plays a fundamental role, and should always be prepared for this diagnostic possibility.]

\section{REFERENCES}

1. Krathen RA, Orengo IF, Rosen T. Cutaneous metastasis: a meta-analysis of data. South Med J. 2003;96:164-7.

2. Rosen T. Cutaneous metastases. Med Clin North Am. 1980 Sep;64:885-900.

3. Hu SC, Chen GS, Lu YW, Wu CS, Lan CC. Cutaneous metastases from different internal malignancies: a clinical and prognostic appraisal. J Eur Acad Dermatol Venereol. 2008;22:735-40.

4. Lookingbill DP, Spangler N, Sexton FM. Skin involvement as the presenting sign of internal carcinoma. A retrospective study of 7316 cancer patients. J Am Acad Dermatol. 1990;22:19-26.

5. Lookingbill DP, Spangler N, Helm KF. Cutaneous metastases in patients with metastatic carcinoma: a retrospective study of 4020 patients. J Am Acad Dermatol. 1993;29:228-36.

6. Schwartz RA. Cutaneous metastatic disease. J Am Acad Dermatol. 1995;33:161-82.

7. Hu SC, Chen GS, Wu CS, Chai CY, Chen WT, Lan CC. Rates of cutaneous metastases from different internal malignancies: experience from a Taiwanese medical center. J Am Acad Dermatol. 2009;60:379-87.

8. Schwartz RA, Rothenberg J. Metastatic adenocarcinoma of breast within a benign melanocytic nevus in the context of cutaneous breast metastatic disease. J Cutan Pathol. 2010;37:1251-4.

9. Brownstein MH, Helwig EB. Patterns of cutaneous metastasis. Arch Dermatol. 1972;105:862-8.

10. Schoenlaub P, Sarraux A, Grosshans E, Heid E, Cribier B. Survival after cutaneous metastasis: a study of 200 cases. Ann Dermatol Venereol. 2001;128:1310-5.

\author{
MAILING ADDRESS: \\ Verônica Riquet de Siqueira \\ Rua Capitão Francisco Pedro, 1290 \\ Rodolfo Teófilo \\ 60430-372 - Fortaleza - CE \\ Brazil \\ E-mail: veronicariquet@yahoo.com.br
}

How to cite this article: Siqueira VR, Frota AS, Maia IL, Queiroz HMC, Valença Júnior JT, Accioly Filho JW. Cutaneous involvement as the initial presentation of metastatic breast adenocarcinoma: a case report. An Bras Dermatol. 2014;(6):960-3. 